SNUTP-99-039

\title{
Normalization Constants of Large Order Behavior
}

\author{
Taekoon Lee* \\ Center for Theoretical Physics \\ Seoul National University \\ Seoul 151-742, Korea
}

\begin{abstract}
A perturbation scheme is discussed for the computation of the normalization constant of the large order behavior arising from an ultraviolet renormalon. In this scheme the normalization constant is expressed in a convergent series that can be calculated using the ordinary perturbative expansion and the nature of the renormalon singularity.
\end{abstract}

PACS numbers: 11.15.Bt, 11.10.Jj, 11.25.Db

Keywords: renormalon, large order behavior

*email: tlee@ctp.snu.ac.kr 
The large order behavior in field theories arising from a renormalon is generally given in the form

$$
a_{n}=K n ! n^{\nu} b_{0}^{-n}[1+O(1 / n)] \quad \text { for } \quad n \rightarrow \infty
$$

While the constants $\nu$ and $b_{0}$ are calculable, the normalization constant $K$ cannot be determined exactly. An infinite number of renormalon diagrams contribute to it, but it is not known how to sum such diagrams to all order [1, 2, 3].

Though the normalization constant cannot be determined exactly, we shall see that it can be calculated perturbatively to an arbitrary precision. The large flavor $\left(N_{f}\right)$ expansion is often invoked for an approximate evaluation of the normalization, and in the literature it is further asserted that the normalization cannot be computed without resorting to it [4]. However, the $N_{f}$ expansion may not be considered a systematic perturbation scheme, as it is not proven that it gives a convergent series or even compatible with nonabelian gauge theories. At large $N_{f}$, the asymptotic freedom is lost, and so it is probably incompatible with asymptotic free gauge theories.

We note, however, that there is a systematic method that is both compatible with nonabelian gauge theories and gives a convergent series. In [1] we have discussed a scheme which express the normalization constant of an infrared (IR) renormalon in a convergent series which depends only on the strength of the renormalon singularity and the ordinary perturbative coefficients of the amplitude in consideration. A sample calculation in QCD using the radiative calculations up to three loops shows that our method gives a rather quickly convergent series. The purpose of this letter is to extend the scheme to the case of an UV renormalon.

Let us first review the perturbation scheme briefly in the case of an IR renormalon. To be specific, we consider a Green's function $D(\alpha)$, such as the Adler function, in QCD and its expansion

$$
D(\alpha)=\sum_{n=0}^{\infty} a_{n} \alpha^{n+1}
$$

The Borel transform $\tilde{D}$ is then defined as follows. We first define it in the neighborhood of the origin as

$$
\tilde{D}(b)=\sum_{n=0}^{\infty} \frac{a_{n}}{n !} b^{n}
$$


and then by analytically continuing to the whole $b$-plane. $\tilde{D}(b)$ is known to have IR renormalon singularities at $b=-n / \beta_{0}, n=2,3,4, \cdots$ and UV renormalon singularities at $b=n / \beta_{0}, n=1,2,3, \cdots$, where $\beta_{0}$ is the first coefficient of the $\beta$-function. Throughout this letter we assume there are no other singularities associated with $\tilde{D}(b)$ except for those caused by the instantons which are irrelevant for our discussion. Now consider the first IR renormalon at $b=-2 / \beta_{0}$. The nature of the first IR renormalon singularity is given in the form [5]

$$
\tilde{D}(b) \approx \frac{\hat{D}}{\left(1+\frac{\beta_{0} b}{2}\right)^{1+\nu}} \quad \text { for } \quad b \approx \frac{-2}{\beta_{0}},
$$

where $\nu=-2 \beta_{1} / \beta_{0}^{2}$, and $\beta_{1}$ is the second coefficient of the $\beta$-function. Then the large order behavior caused by the renormalon is given in the form (11) with $K=\hat{D} / \nu$ ! and $b_{0}=-2 / \beta_{0}$. Note that the normalization constant becomes the residue of the singularity in the $b$-plane. Thus we can equivalently work with the renormalon residue in order to study the normalization constant.

The perturbative calculation of the residue is based on the observation that the residue of the closest singularity to the origin in the complex plane can be expressed in a convergent series involving only the Taylor expansion at the origin. Assuming that the strength of the singularity is known all one needs to calculate the residue is to move the singularity in consideration by conformal mapping closer to the origin than any other singularities in the Borel plane.

This scheme works as follows in the case of the first IR renormalon. The closest singularity to the origin in the $b$-plane is the UV renormalon at $b=1 / \beta_{0}$. Using the conformal mapping

$$
z=-\frac{\beta_{0} b}{1-\beta_{0} b}
$$

we can make the first IR renormalon the closest singularity to the origin. In the $z$-plane the first IR renormalon assumes

$$
\tilde{D}(b(z)) \approx \frac{\left(\frac{2}{9}\right)^{1+\nu} \hat{D}}{\left(\frac{2}{3}-z\right)^{1+\nu}} \quad \text { for } \quad z \approx \frac{2}{3} .
$$

Now consider a function defined by

$$
R(z)=\tilde{D}(b(z))\left(\frac{2}{3}-z\right)^{1+\nu} .
$$


At the IR renormalon singularity, $R(z)$ could still be singular, but it is bounded. The residue is then given by

$$
\left(\frac{2}{9}\right)^{1+\nu} \hat{D}=\left.R(z)\right|_{z=\frac{2}{3}} .
$$

Since $R(z)$ is analytic on the disk $|z|<2 / 3$ we can write (8) in a series form by expanding it at $z=0$

$$
\left(\frac{2}{9}\right)^{1+\nu} \hat{D}=\left.\sum_{n=0}^{\infty} r_{n} z^{n}\right|_{z=\frac{2}{3}} .
$$

It is easy to see that $r_{n}$ depends only on $a_{i}$ with $i \leq n$, and thus calculable.

One might question the convergence of the series (9), since it is evaluated at the renormalon singularity at which $R(z)$ could be singular. However, it should be noted that the finiteness of $R(z)$ at the singularity guarantees the convergence. A numerical evaluation of the series using the 3-loop calculation of the Adler function in QCD shows a quick convergence for small $N_{f}$ case [1]. For example, the first three elements of the series are $0.904,-0.358,0.003$ for $N_{f}=2$, and $0.946,-0.354,-0.098$ for $N_{f}=3$.

This example demonstrates that a renormalon residue can be expressed in a convergent series once the nature of the singularity is known. We now show that a similar conclusion can be made with a UV renormalon. In the following we shall assume that $\beta_{0}<0$, for definiteness, and focus exclusively on the first UV renormalon since it gives the dominant large order behavior. The structure of the UV renormalon is a little more complicated. According to Parisi, it is determined by an insertion of $\operatorname{dim}=6$ operators $[6]$. To be specific, let us consider a Green's function $A(\alpha)$ and its Borel transform $\tilde{A}(b)$. Generally the Borel transform has a branch cut beginning at the first UV renormalon on the negative real axis, and a quantity defined by

$$
\operatorname{Im} A(\alpha)=-\lim _{\epsilon \rightarrow 0} \frac{1}{2 i} \int_{-\infty}^{0} d b e^{-\frac{b}{\alpha}}[\tilde{A}(b+i \epsilon)-\tilde{A}(b-i \epsilon)]
$$

is nonvanishing. The Parisi ansatz states that the dominant contribution to $\operatorname{Im} A(\alpha)$, for $\alpha \rightarrow 0_{-}$, arises from an insertion of $\operatorname{dim}=6$ operators, and is given in the form,

$$
\operatorname{Im} A(\alpha)=\sum_{i=1}^{M} f_{i}(\alpha) O_{i}(\alpha)+O\left(e^{-\frac{2}{\beta_{0} \alpha}}\right)
$$


where the index $i$ runs over all $\operatorname{dim}=6$ operators $O_{i}$. And $f_{i}$ satisfy the renormalization group equation

$$
\left[\left(\beta(\alpha) \frac{d}{d \alpha}-1\right) \delta_{i j}-\gamma_{i j}(\alpha)\right] f_{j}(\alpha)=0
$$

where $\gamma_{i j}$ denotes the anomalous dimension of the associated dim $=6$ operators. An explicit implementation of the Parisi ansatz may be found in [7]. Solving the RG equation one can write formally

$$
\operatorname{Im} A(\alpha)=-\sum_{i}^{M} \frac{\pi \beta_{0} K_{i}}{\Gamma\left(\nu_{i}\right)} e^{-\frac{1}{\beta_{0} \alpha}}\left(\frac{\alpha}{\beta_{0}}\right)^{1-\nu_{i}}\left[1+\sum_{j=1}^{\infty} \frac{\Gamma\left(\nu_{i}\right) C_{i j}}{\Gamma\left(\nu_{i}-j\right)}\left(\frac{\alpha}{\beta_{0}}\right)^{j}\right]+O\left(e^{-\frac{2}{\beta_{0} \alpha}}\right)
$$

where $K_{i}$ are undetermined constants while $\nu_{i}$ depend on both $\beta_{0}, \beta_{1}$ and the one loop anomalous dimension, and $C_{i j}$ are calculable constants depending on the higher order corrections on $\beta(\alpha), \gamma(\alpha)$ and $O_{i}(\alpha)$. Note that the summation within the bracket is not well defined; but, this point will be irrelevant in the following discussion.

The corresponding Borel transform to (13) is then given by

$$
\tilde{A}(b) \approx \sum_{i}^{M} K_{i}\left(1-\beta_{0} b\right)^{-\nu_{i}}\left[1+\sum_{j=1}^{\infty} C_{i j}\left(1-\beta_{0} b\right)^{j}\right]
$$

in the neighborhood of the singularity at $b=1 / \beta_{0}$, and the corresponding large order behavior is given in the form

$$
a_{n}=\sum_{i}^{M} \frac{K_{i}}{\Gamma\left(\nu_{i}\right)} n ! n^{\nu_{i}-1} \beta_{0}^{n}[1+O(1 / n)] .
$$

Our aim is to express the prefactors $K_{i}$ in a calculable, convergent series. Without losing generality, we may assume $\nu_{i}>\nu_{j}$ for $i<j$. And also, for the moment we shall assume all $\nu_{i}>0$, and later will make a comment on the case with a negative $\nu_{i}$. Then it is straightforward to write $K_{1}$, the prefactor of the most dominant term in the large order behavior, in a convergent series. Since

$$
K_{1}=\left.\tilde{A}(b)\left(1-\beta_{0} b\right)^{\nu_{1}}\right|_{b=\frac{1}{\beta_{0}}}
$$

we can obtain a series expression for $K_{1}$ by expanding the function on the r.h.s. at $b=0$. The resulting series is then convergent because the function is finite at the singularity and there is no other singularity within the disk $|b| \leq-1 / \beta_{0}$. 
For the prefactors other than $K_{1}$ we get a linear relation among them. Using (14) it is easy to write $K_{i}$ as

$$
K_{i}=\left.\left[h_{i}(b)+\sum_{j} m_{i j}(b) K_{j}\right]\right|_{b=\frac{1}{\beta_{0}}}
$$

where

$$
h_{i}(b)=\tilde{A}(b)\left(1-\beta_{0} b\right)^{\nu_{i}}
$$

and

$$
m_{i j}=\left\{\begin{array}{l}
-\left(1-\beta_{0} b\right)^{\nu_{i}-\nu_{j}}\left[1+\sum_{k=1}^{\left[\nu_{j}-\nu_{i}\right]} C_{j k}\left(1-\beta_{0} b\right)^{k}\right], \text { for } i>j \\
0, \quad \text { for } i \leq j,
\end{array}\right.
$$

with $\left[\nu_{j}-\nu_{i}\right]$ being an integer satisfying

$$
0 \leq \nu_{j}-\nu_{i}-\left[\nu_{j}-\nu_{i}\right]<1
$$

To solve eq. (17) we introduce $r_{i}(b)$ defined by

$$
r_{i}(b)=\sum_{j}\left[\delta_{i j}-m_{i j}(b)\right] K_{j}-h_{i}(b) .
$$

Note that by definition $r_{i}(b)$ vanishes at the renormalon singularity. From (21) we obtain

$$
K_{i}=\sum_{j}\left[1-m^{(N)}\right]_{i j}^{-1}\left(h_{j}^{(N)}+r_{j}^{(N)}\right)
$$

where $f^{(N)}$ for a function $f(b)$ denotes the $N$-th order Taylor expansion evaluated at the singularity, i.e,

$$
f^{(N)}=\sum_{n=0}^{N} \frac{d^{n} f(0)}{d b^{n}} \frac{\beta_{0}^{-n}}{n !} .
$$

Note that $h_{i}^{(N)}$ is calculable in terms of the perturbative coefficients of $\tilde{A}(b)$. From the definition (19) we have

$$
m_{i j}^{(N)}= \begin{cases}-\frac{N^{\nu_{j}-\nu_{i}}}{\Gamma\left(\nu_{j}-\nu_{i}+1\right)}\left[1+\sum_{k=1}^{\left[\nu_{j}-\nu_{i}\right]} \frac{C_{j k} \Gamma\left(\nu_{j}-\nu_{i}+1\right)}{\Gamma\left(\nu_{j}-\nu_{i}-k+1\right)} N^{-k}\right], & \text { for } i>j \\ 0, & \text { for } i \leq j\end{cases}
$$

and from (14), (21)

$$
\begin{aligned}
r_{i}^{(N)}= & -\sum_{j=1}^{i-1} \sum_{k=\left[\nu_{j}-\nu_{i}\right]+1}^{\infty} \frac{K_{j} C_{j k}}{\Gamma\left(\nu_{j}-\nu_{i}-k+1\right)} N^{\nu_{j}-\nu_{i}-k} \\
& -\sum_{j=i+1}^{M} \frac{K_{j}}{\Gamma\left(\nu_{j}-\nu_{i}+1\right)} N^{\nu_{j}-\nu_{i}}\left[1+\sum_{k=1}^{\infty} \frac{C_{j k} \Gamma\left(\nu_{j}-\nu_{i}+1\right)}{\Gamma\left(\nu_{j}-\nu_{i}-k+1\right)} N^{-k}\right] \\
& +c_{i} N^{-\nu_{i}}(1+O(1 / N))
\end{aligned}
$$


where $c_{i}$ denotes a constant. The terms proportional to $c_{i}$ arise from the regular part of the Borel transform and are not generally calculable. As expected $r_{i}^{(N)}$ vanishes in the large $N$ limit.

Since $m_{i j}^{(N)}$ can be divergent under large $N$ limit, $\sum_{j}\left(1-m^{(N)}\right)_{i j}^{-1} r_{j}^{(N)}$ can be nonvanishing in large $N$ limit, and thus we may write part of (22) as

$$
\sum_{j}\left[1-m^{(N)}\right]_{i j}^{-1} r_{j}^{(N)}=\sum_{j}\left(\Delta_{1}\right)_{i j} K_{j}+r_{i}^{1}
$$

where we have separated those terms nonvanishing under large $N$ limit from those vanishing, and put the former into $\sum_{j}\left(\Delta_{1}\right)_{i j} K_{j}$ and the latter into $r_{i}^{1}$. Note that the nonvanishing part is linear in $K_{i}$. This is because the terms proportional to $c_{i}$ in (25) give rise to a contribution only of $O\left(N^{-\nu_{i}}\right)$, thus affecting only $r_{i}^{1}$, which can be easily seen from the fact that $\left(1-m^{(N)}\right)_{i j}^{-1}$ is at most as divergent as $m_{i j}^{(N)}$ in the large $N$ limit. Substituting (26) into (22) we obtain

$$
K_{i}=\sum_{j}\left[\left(1-\Delta_{1}\right)^{-1}\left(1-m^{(N)}\right)^{-1}\right]_{i j} h_{j}^{(N)}+\sum_{j}\left(1-\Delta_{1}\right)_{i j}^{-1} r_{j}^{1}
$$

We can now repeat this step by

$$
\sum_{j}\left(1-\Delta_{m-1}\right)_{i j}^{-1} r_{j}^{m-1}=\sum_{j}\left(\Delta_{m}\right)_{i j} K_{j}+r_{i}^{m}
$$

for a finite number of times (say $l$ times) until $\sum_{j}\left(1-\Delta_{l}\right)_{i j}^{-1} r_{j}^{l}$ vanishes in the large $N$ limit, to obtain

$$
\vec{K}=\lim _{N \rightarrow \infty}\left[\left(1-\Delta_{l}\right)^{-1} \cdots\left(1-\Delta_{1}\right)^{-1}\left(1-m^{(N)}\right)^{-1} \vec{h}^{(N)}\right]
$$

This is our main result.

Now as an example, let us consider a Borel transform whose UV renormalon singularity is given by (14) with $M=2$. Then from (24) and (25)

$$
m_{21}^{(N)}=-\frac{N^{\nu_{1}-\nu_{2}}}{\Gamma\left(\nu_{1}-\nu_{2}+1\right)}(1+O(1 / N)), \quad \text { otherwise } \quad m_{i j}^{(N)}=0
$$

and

$$
\begin{aligned}
& r_{1}^{(N)}=-\frac{K_{2}}{\Gamma\left(\nu_{2}-\nu_{1}+1\right)} N^{\nu_{2}-\nu_{1}}(1+O(1 / N))+c_{1} N^{-\nu_{1}}(1+O(1 / N)) \\
& r_{2}^{(N)}=-\frac{K_{1} C_{11}}{\Gamma(\delta+1)} N^{\delta}(1+O(1 / N))+c_{2} N^{-\nu_{2}}(1+O(1 / N))
\end{aligned}
$$


where $\delta=\nu_{1}-\nu_{2}-\left[\nu_{1}-\nu_{2}\right]-1<0$. Using the definition (26) we find

$$
\Delta_{1}=\left(\begin{array}{cc}
0 & 0 \\
0 & \frac{1}{\Gamma\left(\nu_{1}-\nu_{2}+1\right) \Gamma\left(\nu_{2}-\nu_{1}+1\right)}
\end{array}\right),
$$

and obtain

$$
\left(\begin{array}{l}
K_{1} \\
K_{2}
\end{array}\right)=\lim _{N \rightarrow \infty}\left[\left(1-\Delta_{1}\right)^{-1}\left(1-m^{(N)}\right)^{-1}\left(\begin{array}{c}
h_{1}^{(N)} \\
h_{2}^{(N)}
\end{array}\right)\right] .
$$

Now having given the series expression for the normalization constants, a comment is in order. In deriving (29) we have assumed that all $\nu_{i}>0$. As long as the normalization constants associated with positive $\nu_{i}$ only are concerned, (29) can be used without modification. However, if some of $\nu_{i}$ are negative, we have to consider $\tilde{A}^{(p)}(b)$ instead of $\tilde{A}(b)$ defined as

$$
\begin{aligned}
\tilde{A}^{(p)}(b) & =\frac{d^{p}}{d b^{p}} \tilde{A}(b) \\
& \approx \sum_{i}^{M} K_{i}^{\prime}\left(1-\beta_{0} b\right)^{-\tilde{\nu}_{i}}\left[1+\sum_{j=1}^{\infty} C_{i j}^{\prime}\left(1-\beta_{0} b\right)^{j}\right] \quad \text { for } \quad b \approx \frac{1}{\beta_{0}}
\end{aligned}
$$

such that $p$ satisfies $\tilde{\nu}_{i}=\nu_{i}+p>0$ for all $\nu_{i}$. Clearly $K_{i}$ are related linearly with $K_{i}^{\prime}$, and so calculable once $K_{i}^{\prime}$ are known. Now $K_{i}^{\prime}$ from $\tilde{A}^{(p)}(b)$ can be obtained in a series form using the steps taken in (17) - (29), and is given by (29) with $\vec{K} \rightarrow \overrightarrow{K^{\prime}}, \nu_{i} \rightarrow \tilde{\nu}_{i}$ and $\tilde{A}(b) \rightarrow \tilde{A}^{(p)}(b)$.

In conclusion, we have shown that the normalization constants of the large order behavior caused by an UV renormalon can be expressed, as in the case of an IR renormalon, in a calculable, convergent series, and thus can be computed to an arbitrary precision using the ordinary weak coupling expansion. Considering that the calculation of the normalization constants is equivalent to summing all sort of the higher order renormalon diagrams, it is surprising that they can be computed from the usual perturbation expansion.

Acknowledgements: This work was supported in part by the Korean Science and Engineering Foundation (KOSEF). 


\section{References}

[1] T. Lee, Phys. Rev. D 56 (1997) 1091.

[2] G. Grunberg, Phys. Lett. B 304 (1993) 183.

[3] M. Beneke and V.I. Zakharov, Phys. Lett. B (1993) 340.

[4] M. Beneke, hep-ph/9807443, and references therein.

[5] A.H. Mueller, Nucl. Phys. B 250 ( 1985) 327.

[6] G. Parisi, Phys. Lett. B 76 (1978) 65.

[7] M. Beneke, V.M. Braun and N. Kivel, Phys. Lett. B 404 (1997) 315. 